\title{
Le statut social des enseignants français
}

$\mathrm{Au}$ prisme du renouvellement générationnel

Géraldine Farges

\section{CpenEdition}

\section{Journals}

Édition électronique

URL : https://journals.openedition.org/ress/884

DOI : $10.4000 /$ ress.884

ISSN : 1663-4446

Éditeur

Librairie Droz

\section{Édition imprimée}

Date de publication : 15 octobre 2011

Pagination : 157-178

ISBN : 978-2-600-01551-6

ISSN : 0048-8046

Référence électronique

Géraldine Farges, "Le statut social des enseignants français », Revue européenne des sciences sociales [En ligne], 49-1 | 2011, mis en ligne le 01 janvier 2015, consulté le 16 février 2023. URL : http:// journals.openedition.org/ress/884; DOI : https://doi.org/10.4000/ress.884 


\title{
LE STATUT SOCIAL \\ DES ENSEIGNANTS FRANÇAIS
}

\section{AU PRISME DU RENOUVELLEMENT GÉNÉRATIONNEL}

\author{
GÉRALDINE FARGES
}

Observatoire Sociologique du Changement - IEP de Paris

geraldinefarges@gmail.com

\begin{abstract}
Résumé. Cet article analyse les transformations du statut social des enseignants en France, en s'interrogeant sur les conséquences des nombreux départs à la retraite qui caractérisent le début du $X X l^{e}$ siècle, et des recrutements consécutifs de «nouveaux enseignants». La valeur sociale des professions de l'enseignement, le niveau d'études, la profession du conjoint et la profession du père sont principalement étudiés, à l'appui de données statistiques. Les éléments que nous présentons témoignent d'évolutions contraires, en chiasme, dans le statut social des enseignants. Nous montrons que les cohortes les plus jeunes bénéficient d'un «prestige» social et intellectuel moindre que les précédentes, alors même qu'elles comptent davantage d'enfants d'enseignants, professions intermédiaires, cadres ou professions libérales en leur sein. Par ailleurs, nous repérons que les comportements conjugaux des plus jeunes enseignants se distinguent de ceux de leurs aînés par une stabilité, voire un rebond, de la dynamique homogame, qui pourrait s'interpréter comme le signe d'une certaine fermeture sur lui-même du groupe enseignant.
\end{abstract}

Mot-clés: enseignants, classes moyennes, générations, statut social, niveau d'études, origine sociale, homogamie

\begin{abstract}
This article analyses French primary and secondary-education teachers' social status, questioning the consequences of the massive retirement movement, and the parallel arrival of "new teachers", that characterize the beginning of the XXI century. Four elements are taken into account: the social value of the teaching profession, the educational level, the professional activity of teachers' spouse, and their social origin. Different data are used. We demonstrate that teachers' social status has changed following opposite dynamics: on the one hand, the social and intellectual "prestige" of their professional activity has declined, on the other hand, they more often originate from middle and superior social categories. Besides we point out that the matrimonial behaviors of the youngest teachers tend to be more concerned by social homogamy than their predecessors', what suggests a certain form of social closing.
\end{abstract}

Keywords: teachers, middle classes, generational change, social status, educational level, social origin, social homogamy 
En France, l'étude de la population enseignante est chargée d'implications sociales actuellement: sur une courte période, des années 1950 au milieu des années 1970, l'Éducation nationale a recruté massivement des jeunes enseignants qui ont représenté la plus importante proportion de ses personnels jusqu’à leur départ à la retraite, dans les années 2000. Dès lors, ce sont des cohortes de jeunes enseignants qui viennent remplacer ceux qui ont débuté quarante ans auparavant, même si les mouvements d'effectifs sont plus complexes dans le détail'. Cette dynamique démographique saccompagne de transformations qui s'étendent au-delà des considérations quantitatives. En effet, elle se produit dans un contexte à l'embauche sensiblement renouvelé. Louis Chauvel utilise l'expression de «socialisation transitionnelle» pour caractériser la période située entre la fin des études et la stabilisation de l'âge adulte, et durant laquelle se joue l'avenir des individus : «en peu de temps, quelques mois en général, les potentialités conférées par la famille et l'éducation se transforment en réalités concrètes sur la base desquelles les gens construisent leurs trajectoires de vie » (2006a, p. I6I). Ainsi, le processus de «socialisation transitionnelle» suppose que les individus conservent durablement l'empreinte de la situation sociale, économique, culturelle ou politique rencontrée au moment de l'entrée dans la vie adulte. Et cet effet est d’autant plus marqué pour les populations, comme celle des enseignants, qui supposent à la fois peu de mobilité interprofessionnelle au cours de la vie active et des recrutements par «vagues», sans véritables perspectives de long terme (Chapoulie, 1987 ; Chauvel, 1998).

I Ce point est développé dans notre thèse (Farges, 2010). En 2007, environ 18000 départs en retraite ont été comptabilisés parmi les enseignants du second degré et assimilés, près de 12000 dans le premier degré, alors que ces effectifs se situaient aux alentours de 11000 pour chaque niveau en 2000; en moyenne sur la période 2000-2007, 12800 départs en retraite par an ont été enregistrés parmi les enseignants du premier degré public, 14700 dans le second degré public (Baraton et Perronnet, 2009). Par ailleurs, en 2007, le premier gouvernement Fillon a annoncé la révision générale des politiques publiques et s'est engagé dans une politique de non-remplacement d'un fonctionnaire sur deux partant à la retraite entre 2009 et 201I (Ministère du Budget, des Comptes publics, et de la Réforme de l'État, RGPP: Quatrième rapport, juin 2010). 
On se propose d’analyser ici les transformations du statut social associé au métier d'enseignant, c'est-à-dire aux modifications dans le temps de ce que signifie être enseignant dans la société française. Cette perspective emprunte à la sociologie de Max Weber, qui réalise l'étude des groupes sociaux à la fois sur les conditions objectives d'existence des individus et sur leur ständiche Lage (Weber, I976 [1922], p. I79) (que nous traduisons par «rang» ou «statut», comme le suggère JeanPierre Grossein (2005)), c'est-à-dire le «privilège positif ou négatif de considération sociale» fondé sur le mode de vie, le type d'instruction, le prestige de naissance ou le prestige de la profession (Weber, I995 [1956], p. 395) : dans quelle mesure les jeunes enseignants qui entament leur carrière depuis le début des années 2000 partagent-ils le même statut que leurs aînés, quittant progressivement la vie active?

Nous poursuivons le double objectif d'analyser les transformations du statut social des enseignants et de mettre en évidence les différences générationnelles qui parcourent actuellement cette population. Les personnels du premier et du second degré sont étudiés conjointement, et nous formulons l'hypothèse selon laquelle leurs statuts connaissent un rapprochement, au-delà de celui quá impulsé la loi d'orientation sur l'éducation du ıo juillet $1989^{2}$. Suivant l'approche weberienne, nous mobilisons quatre indicateurs du statut social : la valeur sociale de la profession d'enseignant, le niveau d'études, la profession du conjoint, ainsi que le milieu social d'origine.

\section{LA VALEUR SOCIALE D’UN MÉTIER}

Par valeur sociale, nous comprenons le «privilège de considération sociale» dont les enseignants bénéficient par le «prestige» particulier de leur profession. Il est malaisé d'identifier le prestige d'une profession (Lemel, I99I ; Chambaz, Maurin et Torelli, I998), néanmoins nous tenterons de l’approcher en considérant les représentations sociales associées au métier d'enseignant. Celui-ci, pour le premier comme

2 Cette loi concrétise l'unification des parcours de formation entre premier et second degrés par la création des IUFM (Instituts Universitaires de Formation des Maîtres). Le décret du |er août 1990 crée le corps des «professeurs des écoles», qui est classé en catégorie A de la fonction publique, au même titre que les professeurs certifiés, alors que le corps des instituteurs était classé en catégorie B. 
pour le second degré, est considéré comme ayant perdu de sa valeur depuis la seconde moitié du $\mathrm{Xx}^{\mathrm{e}}$ siècle. Cependant, il est possible de distinguer deux périodes.

La première s'étend des années 1950 au début de la décennie 1980. Comme l’a écrit Henri Mendras (1994), les enseignants sont alors, avec les autres membres des «nouvelles classes moyennes salariées», un des principaux moteurs du changement social, de la «Seconde Révolution française». Entre 1969 et 1975, les effectifs de la population enseignante ont progressé de $26 \%$, passant de 560000 individus à 700000 environ33. Jusquau milieu des années 1970, instituteurs et professeurs acquièrent une importance numérique inégalée jusqu’alors, en absolu comme en proportion de la population active. Dans un contexte de volontarisme politique et de fortes attentes sociales à l'égard de la scolarisation, cet accroissement apporte une réponse à l'augmentation des effectifs scolaires (Prost, 2004 [1981]). Toutefois, s'il s'agit bien d'une dynamique sociale d'ensemble, cela ne signifie pas que les professions de l'enseignement jouissent en elles-mêmes d'un important prestige. En 1957, Ida Berger, dans un article portant sur «le malaise des instituteurs», fait état d'une «doléance exprimée avec vigueur par un très grand nombre d'instituteurs et d'institutrices [qui] concerne la perte du prestige social de leur profession» (1957, p. 337). De même, Jean-Michel Chapoulie et Dominique Merllié évoquent une «dévalorisation relative, sous le rapport de la valeur sociale, de la profession enseignante, corrélative des difficultés de recrutement» (197I, p. 62). En fait, les représentations sociales associées au métier d’enseignant se sont transformées, avec la construction d'un système scolaire unifié. Dès lors, enseigner dans le premier ou dans le second degré, perçus comme des jalons conduisant aux études supérieures, est moins valorisé, d’autant plus que l’allongement de la scolarité au sein de la population active a décuplé les débouchés professionnels alternatifs des jeunes diplômés. Il n’en demeure pas moins que la fin des années 1970 et le début des années 1980 correspondent à la plus forte présence des enseignants parmi les hommes et les femmes politiques au $\mathrm{xx}^{\mathrm{e}}$ siècle, ce qui témoigne du rôle social qu’ils jouent à cette époque. Leur représen-

3 Source: Enquêtes Emploi 1969 et 1975 (microdonnées fournies par le Centre Maurice Halbwachs, calculs de l'auteur), enseignants (repérés à l'aide du code détaillé des professions, sans distinction public-privé) actifs âgés de 18 à 64 ans, effectifs extrapolés à l'aide des variables fournies. Précisons, à l'aide de ces mêmes sources, que l'accroissement est plus important dans le secondaire (+65\%) que dans le primaire $(+15 \%)$. 
tation politique dépasse alors de loin leur poids dans la population active: en 198I, les professeurs du supérieur représentent $8,2 \%$ de l'Assemblée Nationale, les professeurs du second degré II, $3 \%$, les instituteurs $4,7 \%$; la moitié des nouveaux élus en I98I sont enseignants (Collovald, I985; Sawicki, 2008).

La deuxième période va du milieu des années 1980 aux années 2000. Les années I980 signent, selon Henri Mendras, la fin de la «Seconde Révolution française», les difficultés économiques ayant raison de la dynamique culturelle portée par les classes moyennes (Chauvel, 2006b). L'espoir de promotion sociale via l'école, nourri par les familles, s'émousse avec le chômage persistant ${ }^{4}$, ce qui se répercute nécessairement sur ceux qui font l'école, d'autant plus que l'image qui en émane est celle d'une institution en crise, impuissante face à la découverte de l'échec scolaire (Hamon et Rotman, 1984; Plaisance, 1985). Pour l'ensemble de ces raisons, dans les années 1980 les écoles normales se vident, les instituteurs ont un «coup au moral» (Charles, 1988). L'instauration du statut de professeur des écoles en 1990, ainsi que la politique visant à amener $80 \%$ d'une classe d'âge au baccalauréat à l'horizon de l'an $2000^{5}$, donnent une nouvelle dynamique au métier d'enseignant dont le nombre de candidats à l'entrée connaît une certaine croissance sur la fin du Xx siècle, sans devenir un métier particulièrement désirable pour autant (Chambaz, Maurin et Torelli, 1998). En l'occurrence, à l'aide des résultats de l'enquête que nous avons menée en $2008^{6}$, on observe que les enseignants, dans l'ensemble, partagent la même impression selon laquelle leur profession est peu valorisée: quel que soit le niveau d'enseignement et quel que soit leur âge, ils sont en majorité «peu d’accord» et «pas du tout d’accord» avec l'idée selon laquelle ils sont perçus comme ayant un métier source de valorisation sociale. Cependant, ce désaccord est plus marqué parmi les personnels d'âge intermédiaire (35-45 ans) c'est-à-dire ceux qui sont nés entre 1963 et I973 et ont pour la plupart débuté leur carrière à la charnière des années 1980 et 1990 .

4 À l'exception des familles issues de l'immigration, si l'on suit l'analyse proposée par Louis-André Vallet (1996).

5 Déclaration sur les lycées du 12 novembre 1985.

6 Cette enquête a été réalisée dans le cadre de notre doctorat de sociologie, qui en présente de plus amples résultats. II s'agit d'une enquête par questionnaire interrogeant des enseignants du premier et du second degré $(\mathrm{N}=3000)$ sur leurs modes de vie. Elle a été réalisée avec le soutien logistique et financier de la MAIF. 
Tableau I : Opinion sur la valorisation sociale du métier d'enseignant, $\mathrm{I}^{\mathrm{er}}-2^{\text {nd }}$ degrés, selon l'âge

\begin{tabular}{|c|c|c|c|c|c|c|c|c|}
\hline \multirow[b]{2}{*}{$\begin{array}{l}\text { «Dans la société, pensez- } \\
\text { vous que les enseignants } \\
\text { sont perçus comme ayant } \\
\text { un métier source de } \\
\text { valorisation sociale?» }\end{array}$} & \multicolumn{4}{|c|}{ I ER DEGRÉ } & \multicolumn{4}{|c|}{$2^{\mathrm{ND}}$ DEGRÉ } \\
\hline & ENSEMBLE & $\begin{array}{c}+ \text { DE } 45 \\
\text { ANS }\end{array}$ & $\begin{array}{c}35-45 \\
\text { ANS }\end{array}$ & $\begin{array}{c}34 \text { ANS } \\
\&-\end{array}$ & ENSEMBLE & $\begin{array}{c}+ \text { DE } 45 \\
\text { ANS }\end{array}$ & $\begin{array}{c}35-45 \\
\text { ANS }\end{array}$ & $\begin{array}{c}34 \text { ANS } \\
\&-\end{array}$ \\
\hline Tout à fait d'accord & 4,5 & 4,1 & 3,2 & 6,2 & 5,6 & 4 & 6,3 & 6,9 \\
\hline Assez d'accord & 20,7 & 25,3 & 16 & 20,1 & 20,9 & 21,1 & 21,1 & 20,5 \\
\hline Un peu d'accord & 31,6 & 28,8 & 32,7 & 33,6 & 31,4 & 34,5 & 28,5 & 30,5 \\
\hline Pas du tout d'accord & 37,9 & 34,5 & 43,1 & 36,5 & 36,9 & 33,3 & 40,8 & 37,5 \\
\hline Sans opinion & 5,4 & 7,3 & 5 & 3,6 & 5,1 & 7,1 & 3,2 & 4,6 \\
\hline TOTAL & 100 & 100 & 100 & 100 & 100 & 100 & 100 & 100 \\
\hline LIEN STATISTIQUE & \multicolumn{4}{|c|}{$\times 2: 17,2 * *, d d 1: 8$} & \multicolumn{4}{|c|}{$X 2: n s, d d l: 8$} \\
\hline
\end{tabular}

Enquête Enseignants 2008, effectifs pondérés, calculs de l'auteur, champ: population active âgées de 59 ans ou moins pour le second degré, de 56 ans ou moins pour le premier degré . ****: signif<0,00 I; ***: 0,00 I<signif<0,05:*0,05<signif<0, I ; ns: non-significatif.

\section{ÊTRE DIPLÔMÉ DANS UN CONTEXTE D'«INFLATION SCOLAIRE»}

Une des raisons de la baisse de la valeur sociale du statut d'enseignant est liée à l'allongement général du temps d'études. Est-il encore possible de définir le statut social de l'enseignant à l'aide des capitaux scolaires détenus? Si on utilise l'âge moyen d'entrée dans la vie active comme indicateur', on constate que l'écart entre la catégorie des «professeurs, professions scientifiques» (parmi lesquels se trouvent les plus diplômés) et l'ensemble des actifs s'est réduit en quelques décennies: supérieur de près de sept ans en 1970, il est passé à quatre ans et demi en 2003. En effet, l'âge moyen d'entrée dans la vie active s'est élevé pour tous les actifs, mais le gradient entre les dates extrêmes est plus important pour l'ensemble des actifs

7 Cet indicateur apparaît approprié pour caractériser l'allongement du temps d'études, avec lequel on sait qu'il est fortement corrélé (Van de Velde, 2008). II permet une approche plus synthétique des transformations que le niveau d'études, qui supposerait de tenir compte de l'hétérogénéité dans le temps des niveaux de diplômes obtenus par les enseignants. 
hors enseignants (+3,9 ans) que pour les professeurs, professions scientifiques (+r,6 an) et les instituteurs et assimilés ( $+2,5$ ans), même s'il faudrait entrer dans le détail de la composition de la population active pour affiner l'analyse. On peut de surcroît observer un rapprochement selon le niveau d'enseignement : alors que 2,9 ans séparaient en 1985 l'âge moyen d’entrée dans la vie active des agrégés et certifiés, d'une part, et des instituteurs, d'autre part, l'écart s'est réduit à I,3 an en $2003^{8}$.

Figure I : Âge moyen d'entrée dans la vie active, 1970-20039
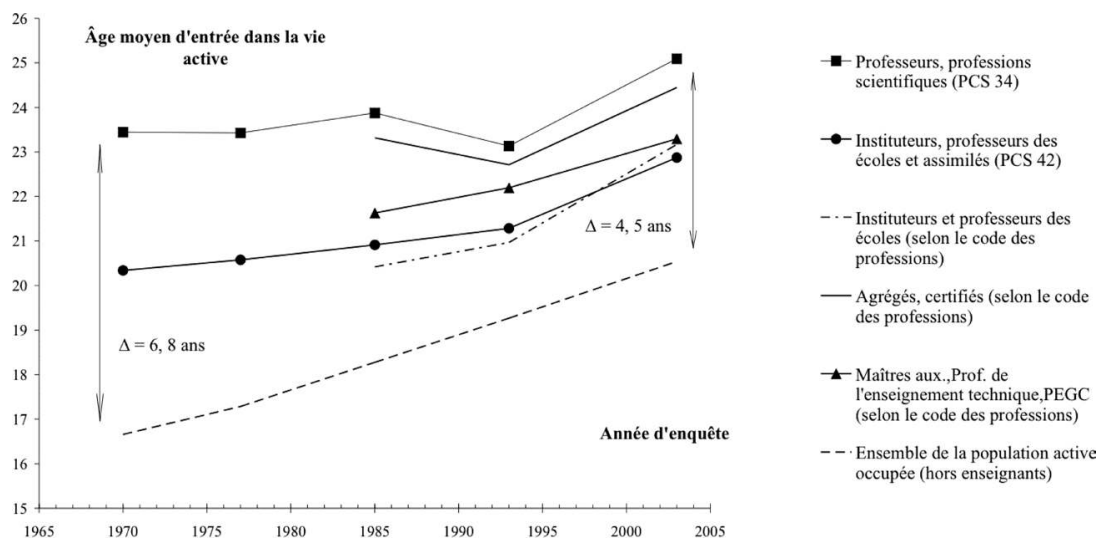

- - Ensemble de la population active occupée (hors enseignants)

Source: Enquêtes Formation et Qualification Professionnelle (INSEE) 1970, 1977, 1985, 1993, 2003 (microdonnées: Centre Maurice Halbwachs), calculs de l'auteur.

8 Dans les années 1990, l'âge moyen d'entrée dans la vie active des professeurs du second degré a baissé, ce qui est vraisemblablement lié à de l'accroissement des besoins d'encadrement consécutif à «la politique des $80 \%$ ».

9 Champ: Population active occupée, âgée de 20 à 64 ans, effectifs pondérés à l'aide des variables fournies. Nomenclatures utilisées: «professeurs, professions scientifiques» = code 32 en 1970, 1977, 34 à partir de 1985; «instituteurs, professeurs des écoles et assimilés 》= code 4 I en 1970, 1977, 42 à partir de 1985. Le code des professions de 1982 a été utilisé, c'est pourquoi les données pour les années 1970 et 1977 ne sont pas disponibles: Notons que les différentes éditions de l'enquête Formation et Qualification Professionnelle mobilisées n'ont pas précisément la même définition du premier emploi, notamment concernant l'inclusion ou l'exclusion de la formation professionnelle, ce qui peut contribuer à élever ou diminuer l'âge d'entrée dans la vie active. 
Pour les jeunes enseignants ayant débuté aux alentours de 1970, le statut de diplômé fait sens. Les individus ont pu se construire autour de cette image d'eux-mêmes, et il est possible de penser que cette image a accompagné leur carrière professionnelle par l'effet durable de la socialisation au métier d'enseignant qu'ils ont connue. En somme, les jeunes enseignants des années I960-1970 ont, au même titre que les générations qui les ont précédés ${ }^{10}$, continué à revêtir une identité sociale et professionnelle d'intellectuels.

Pour les jeunes enseignants qui débutent leur carrière par la suite, une telle construction de soi est moins évidente, bien que leur niveau d'études se soit élevé dans la seconde moitié du $\mathrm{Xx}^{\mathrm{e}}$ siècle, en particulier pour les instituteurs ${ }^{11}$. Les jeunes enseignants entrés dans les années 2000 construisent plutôt une identité professionnelle autour de l'insuffisance de leur formation. Notamment, sur la période récente, les candidats expérimentent la «mastérisation» de leur formation, qui a explicitement pour objectif d'élever le niveau de qualification des personnels enseignants $^{12}$. En pratique, la réforme ne modifie pas leur temps total d'études puisque la plupart des candidats aux métiers de l'enseignement possède de fait un «bac+5 » à l'issue de leur cursus universitaire ${ }^{13}$ et de leur passage en IUFM.

I0 Jusqu'au milieu du $x x^{\mathrm{e}}$ siècle, les professeurs sont les héritiers de la figure sociale et intellectuelle des universitaires du siècle précédent, entretenant des liens à la fois proches et distants (dans la mesure où ils sont responsables de l'éducation de leurs enfants) avec les élites économiques, politiques et religieuses (Gerbod, 1965; Durkheim, 1999 [1938]; Savoie, 2009). Instituteurs et institutrices, titulaires du brevet supérieur et d'une formation à la sortie de l'école normale, incarnent la promotion sociale par l'école promise par la Troisième République (Delsaut, 1992).

II En 1979, leur formation s'est ouverte à l'université, et la loi d'orientation sur l'éducation du 10 juillet 1989, ainsi que le décret du ler août 1990 relatif au statut des professeurs des écoles, ont instauré le niveau licence plus une année de formation pédagogique dans un IUFM (la plupart du temps précédée d'une année de préparation au concours) comme norme d'accès à la profession.

12 La «mastérisation» désigne la réforme de la formation des enseignants proposée par le gouvernement en octobre 2008 et ayant pour objectifs d'élever le niveau de qualification des personnels enseignants, d'intégrer la formation des maîtres dans le dispositif «L.M.D.», de préserver les possibilités de réorientation pour les étudiants non recrutés, de préparer progressivement au métier avant les concours, d'offrir des mécanismes d'encouragement et de promotion sociale (selon l'information du 6/01/20l0 du Ministère de l'Éducation nationale).

13 À la session de recrutement de 2006, près d'un quart des admis au concours de professeur des écoles disposait d'un niveau d'études supérieur à la licence requise (18,6\% étaient titulaires d'un bac +4, 4, l\% d'un bac+5) (Michel, 2007). Dans le second degré, près de la moitié des admis aux concours externes possédait une maîtrise (48,4\% précisément) et 10,9\% étaient titulaires d'un 
Élever le niveau de formation des enseignants s'inscrit dans une volonté de répondre aux attentes d'une société globalement plus instruite et exigeante vis-àvis de son école, d'autant plus lorsque les diplômes tendent à perdre de leur valeur (Duru-Bellat, 2006). À la session 2009, la proportion de bacheliers dans une génération atteignait $65,8 \%$, alors quelle s'élevait aux alentours de $25 \%$ en 1980 et environ $5 \%$ en $1950^{14}$. Nombre des parents d'élèves sont au moins aussi diplômés que les enseignants ${ }^{15}$, et dans des disciplines qui peuvent apparaître davantage valorisées socialement dans la mesure où elles ouvrent aux fonctions d'encadrement, particulièrement bien évaluées (Chambaz, Maurin et Torelli, 1998). Les parents d'élèves se présentent comme de nouveaux acteurs de l'école, ils font valoir leurs compétences en tant qu’observateurs critiques, et en tant quacteurs de la scolarité de leurs enfants (Ballion, 1982; Poupeau, 2008), ce qui constitue une source de tensions pour les enseignants (Dubet, 2002; Lantheaume et Hélou, 2008).

En somme, alors que le niveau d'études possédé a suffi à poser le statut des enseignants recrutés jusqu’aux années 1970, ceux qui sont arrivés par la suite ont eu à se «mettre à niveau » par rapport aux autres actifs.

\section{LES CARACTÉRISTIQUES SOCIALES DU CONJOINT SELON LE GENRE}

Ces transformations ont-elles les mêmes conséquences pour les hommes et pour les femmes? Nous pouvons apporter des éléments de réponse à cette question en analysant selon le genre la profession du conjoint, qui permet de porter un regard sur l'attractivité sociale des métiers de l'enseignement et informe sur le mode de vie puisque les ressources du ménage (Léger, 1983; Chapoulie, 1987), mais également les comportements quotidiens (Singly (de) et Poissenot, 1996), diffèrent en fonction des caractéristiques du conjoint.

diplôme supérieur ou égal à bac+5, ou diplômés d'une école d'ingénieur (Valette, 2007).

14 «L'Éducation nationale en chiffres-édition 2010》, Ministère de l'Éducation nationale.

15 Notons que 27\% des sortants du système éducatif sur la période 2007-2009 possèdent un DEUG, une licence ou plus, un diplôme de grande école ("L'Éducation nationale en chiffresédition $2010 »$, Ministère de l'Éducation nationale). 
En premier lieu, nous pouvons constater, à l'aide des tableaux ci-dessous, que parmi les enseignants nés dans les années $1970^{16}$, les comportements conjugaux des hommes et des femmes se distinguent : quel que soit le niveau d'enseignement, les premiers se mettent davantage en couple avec des enseignantes, tandis que les femmes sont davantage «hypergames», ayant plus souvent que les hommes des conjoints cadres ou professions libérales. Ces résultats sont conformes aux analyses antérieures menées sur ce sujet, qui précisent aussi qu’au-delà du sexe, les comportements conjugaux varient sensiblement en fonction du statut (agrégé, certifié...) ou de la discipline enseignée (Berger et Benjamin, 1964; Léger, 1983; Chapoulie, 1987; Singly (de) et Poissenot, 1996). Cependant, un regard sur le temps long, par cohortes de naissance, des caractéristiques du conjoint témoigne de dynamiques différentes pour les hommes et les femmes.

L'éventail de choix du conjoint était assez limité jusqu'à la première moitié du $\mathrm{xx}^{\mathrm{e}}$ siècle pour les femmes professeurs de collège et de lycée, se mettre en couple avec un homme de moindre qualification compromettant la situation sociale et intellectuelle acquise (Cacouault-Bitaud, 2007). Or, la proportion d'entre elles partageant la vie d'un homme cadre ou profession libérale s'est réduite au cours des décennies passées. Les institutrices / professeurs des écoles connaissent les comportements les plus stables sur la période étudiée. En revanche, les femmes professeurs de collège et de lycée se mettent davantage en couple avec des hommes exerçant une profession intermédiaire, des employés ou des ouvriers, avec lesquels elles ont davantage de traits communs. En effet, on peut faire l'hypothèse qu'elles ne les «effraient» plus sur le plan symbolique, vraisemblablement parce que la possession de diplômes s'est généralisée, banalisée, dans la société, mais également parce que ces hommes sont eux-mêmes davantage diplômés qu'auparavant.

16 En raison des changements intervenus à partir de 2003 sur les enquêtes Emploi, seules les séries 1982-2002 ont été utilisées. De plus, nous n'avons pas opéré de sélection en fonction de l'âge (il aurait en effet été plus pertinent de ne travailler que sur une même tranche d'âge) de façon à conserver des effectifs suffisamment importants. 
À l'inverse, au fil des cohortes successives, les hommes enseignants se mettent davantage en couple avec des femmes cadres et professions libérales, ainsi qu'avec des femmes exerçant une profession intermédiaire. Certes, les résultats présentés portent les marques des évolutions de structure de la population active féminine, dont il s’agirait de contrôler les effets. Ces résultats «bruts » témoignent du fait que, d’une manière générale, le niveau de vie des ménages auxquels appartiennent les hommes enseignants s'est élevé dans la seconde moitié du $\mathrm{xx}^{\mathrm{e}}$ siècle, contrairement à celui des femmes. Néanmoins, on observe que les transformations les plus marquées des caractéristiques du conjoint s'effectuent entre les cohortes nées en 1940-1949 et 1950-1959, alors que la féminisation de la population enseignante sur cette période aurait pu avoir comme effet d'accroître la proportion d'hommes en couple avec une collègue. Le déclin de l'homogamie, pour les hommes comme pour les femmes, témoigne ainsi d'un certain décloisonnement de la population enseignante, «ghetto sociologique» pour reprendre l'expression employée par Gérard Vincent (1967). Cette évolution est conforme aux hypothèses d'Henri Mendras (1994), pour lequel, dans le contexte de la «Seconde Révolution française», les enseignants font partie de la «constellation centrale» dont les membres ${ }^{17}$ se caractérisent par de nombreux échanges, notamment sur le plan matrimonial.

Et pourtant, force est de constater une autre dynamique parmi les plus jeunes enseignants. Les hommes instituteurs / professeurs des écoles et les femmes professeurs du second degré nés dans les années 1970 présentent des comportements homogames plus marqués que leurs prédécesseurs nés dans les années 1960. Pour les hommes professeurs du second degré ou les femmes institutrices / professeurs des écoles, on observe plutôt une stabilité de la proportion des couples de pairs entre les natifs des années 1960 et ceux des années 1970, alors qu’elle était auparavant en baisse. Cela peut conduire à deux hypothèses. D’une part, cela peut dissimuler un «effet d'âge», car, dans la mesure où nos données ne supposent pas de sélection selon l'âge des individus, la cohorte 1970-1979 est plus jeune que les cohortes précédentes. Cependant, il n'est pas possible d'exclure une hypothèse de transfor-

17 «Cadres supérieurs, ingénieurs, professeurs, professions libérales partagent la même culture et la même façon de vivre et ont une forte propension à l'homogamie» (1994, p. 68). 
mations générationnelles, allant dans le sens d'un renouveau des comportements homogames après plusieurs décennies de déclin. Ce renouveau pourrait notamment s'expliquer par une tendance à l'entre-soi, favorisée par le sentiment d'exercer une profession socialement peu valorisée. En outre, l'élévation de la proportion d’enseignants ayant un père enseignant pourrait favoriser une préférence pour un conjoint exerçant également cette profession.

Tableau 2: Profession de la conjointe des hommes professeurs du second degrét8

\begin{tabular}{|l|c|c|c|c|c|}
\hline & \multicolumn{5}{|c|}{ COHORTE DE NAISSANCE DES HOMMES PROFESSEURS } \\
\hline \multicolumn{1}{|c|}{ PROFESSION DE LA CONJOINTE } & 1930 -1939 & 1940 -1949 & 1950 - 1959 & 1960 -1969 & 1970-1979 \\
\hline Agricultrice, artisane, commerçante, ouvrière & 4,4 & 4,3 & 2,8 & 2,2 & 1,5 \\
\hline Cadre, profession libérale & 8,3 & 8,2 & 12,9 & 14 & 17,1 \\
\hline Professeur du second degré & 35,1 & 35,6 & 28,7 & 22,9 & 22,9 \\
\hline Institutrice/ professeur des écoles & 16,6 & 16,6 & 13,9 & 12 & 14,4 \\
\hline Profession intermédiaire & 16,1 & 17,8 & 20,9 & 26 & 27 \\
\hline Employée TOTAL & 19,5 & 17,6 & 20,6 & 23 & 17 \\
\hline & 100 & 100 & 100 & 100 & 100 \\
\hline
\end{tabular}

I8 Champ: Professeurs du second degré actifs identifiés à l'aide du code des professions (ont été regroupés ici les «professeurs agrégés et certifiés» (34II), les «PEGC et maîtres auxiliaires de l'enseignement général» (422I) et «les enseignants du technique court» (4224)). La profession du conjoint est établie suivant la nomenclature des PCS, sauf pour la catégorie «professeur du second degré» (établie en utilisant le code des professions, regroupant les professeurs agrégés et certifiés, les PEGC et maîtres auxiliaires de l'enseignement général et les enseignants du technique court) et la catégorie «instituteur/professeur des écoles» (établie en utilisant le code des professions, regroupant les instituteurs, les directeurs d'école primaire ou maternelle et les instituteurs de l'éducation spécialisée). Les couples sont ici caractérisés par l'indicateur de la «vie en couple», ils ont été repérés par le partage d'un même identifiant de logement par deux individus de sexe différent classés comme «chef de ménage» ou «conjoint(e)». 
Tableau 3: Profession du conjoint des femmes professeurs du second degrér ${ }^{19}$

\begin{tabular}{|l|c|c|c|c|c|}
\hline & \multicolumn{5}{|c|}{ COHORTE DE NAISSANCE DES FEMMES PROFESSEURS } \\
\hline \multicolumn{1}{|c|}{ PROFESSION DU CONJOINT } & $1930-1939$ & $1940-1949$ & $1950-1959$ & $1960-1969$ & 1970-1979 \\
\hline Agriculteur, artisan, commerçant & 6,5 & 7,3 & 7,4 & 5,8 & 3,3 \\
\hline Cadre, profession libérale & 43,3 & 41 & 35,5 & 32,6 & 22,6 \\
\hline Professeur du second degré & 29,4 & 26,5 & 22 & 19,1 & 22,1 \\
\hline Instituteur/professeur des écoles & 3,6 & 2,1 & 3,3 & 2,7 & 2,8 \\
\hline Profession intermédiaire & 12 & 15,7 & 21,5 & 22 & 28 \\
\hline Employé & 1,9 & 3,7 & 4,7 & 7,2 & 8,4 \\
\hline Ouvrier & 3,2 & 3,6 & 5,6 & 10,5 & 12,9 \\
\hline & 100 & 100 & 100 & 100 & 100 \\
\hline
\end{tabular}

Tableau 4: Profession de la conjointe des hommes instituteurs / professeurs des écoles ${ }^{20}$

\begin{tabular}{|c|c|c|c|c|c|}
\hline \multirow[b]{2}{*}{ PROFESSION DE LA CONJOINTE } & \multicolumn{5}{|c|}{$\begin{array}{l}\text { COHORTE DE NAISSANCE DES HOMMES INSTITUTEURS/PROFESSEURS } \\
\text { DES ÉCOLES }\end{array}$} \\
\hline & $1930-1939$ & 1940-1949 & $1950-1959$ & $1960-1969$ & $1970-1979$ \\
\hline Agricultrice, artisane, commerçante, ouvrière & 2,2 & 5,2 & 1,9 & 0,9 & 0,5 \\
\hline Cadre, profession libérale & 1,2 & 2,3 & 6,3 & 6,8 & 10,5 \\
\hline Professeur du second degré & 10,1 & 8,3 & 8 & 8 & 7,4 \\
\hline Institutrice/professeur des écoles & 52,2 & 47,1 & 45 & 43,6 & 51,7 \\
\hline Profession intermédiaire & $|4|$, & 15,2 & 21,1 & 23 & 17,1 \\
\hline Employée & 20,2 & 21,9 & 17,6 & 17,7 & 12,7 \\
\hline TOTAL & 100 & 100 & 100 & 100 & 100 \\
\hline
\end{tabular}

19 Pour les informations relatives à ces données, voir note 18.

20 Champ: Instituteurs/professeurs des écoles actifs identifiés à l'aide du code des professions (ont été regroupés ici les «instituteurs» (42II), «les directeurs d'école primaire ou maternelle» (42/4), et les «instituteurs de l'éducation spécialisée» (4215)). La profession du conjoint est établie suivant la nomenclature des PCS, sauf pour la catégorie «professeur du second degré» (établie en utilisant le code des professions, regroupant les professeurs agrégés et certifiés, les PEGC et maîtres auxiliaires de l'enseignement général et les enseignants du technique court) et la catégorie «instituteur/professeur des écoles» (établie en utilisant le code des professions, regroupant les instituteurs, les directeurs d'école primaire ou maternelle et les instituteurs de l'éducation spécialisée). Les couples sont ici caractérisés par l'indicateur de la «vie en couple», ils ont été repérés par le partage d'un même identifiant de logement par deux individus de sexe différent classés comme «chef de ménage» ou «conjoint(e)». 
Tableau 5 : Profession du conjoint des femmes institutrices/professeurs des écoles ${ }^{21}$

\begin{tabular}{|l|c|c|c|c|c|}
\hline & \multicolumn{5}{|c|}{ COHORTE DE NAISSANCE DES FEMMES INSTITUTRICES/ PROFESSEURS DES } \\
ÉCOLES \\
\hline \multicolumn{1}{|c|}{ PROFESSION DU cONJOINT } & $1930-1939$ & $1940-1949$ & $1950-1959$ & $1960-1969$ & $1970-1979$ \\
\hline Agriculteur, artisan, commerçant & 9,1 & 10,2 & 8,4 & 6,3 & 3,5 \\
\hline Cadre, profession libérale & 23,9 & 23,9 & 24,1 & 24,8 & 25,7 \\
\hline Professeur du second degré & 11,1 & 13,1 & 8,5 & 6,9 & 7,2 \\
\hline Instituteur/professeur des écoles & 14,8 & 12,7 & 14,8 & 10,4 & 10,1 \\
\hline Profession intermédiaire & 23,3 & 22,8 & 26,1 & 28,3 & 28,7 \\
\hline Employé & 6,6 & 6,6 & 8,1 & 9,9 & 12,2 \\
\hline Ouvrier & 11,3 & 10,6 & 10 & 13,3 & 12,7 \\
\hline & 100 & 100 & 100 & 100 & 100 \\
\hline
\end{tabular}

[Tableaux 2, 3, 4 et 5]. Source: Enquêtes Emploi (INSEE) 1982-2002 (microdonnées: Centre Maurice Halbwachs), calculs de l'auteur, effectifs pondérés.

\section{DAVANTAGE D'«HÉRITIERS »ET DE «DÉCLASSÉS »}

En effet, la proportion d'enseignants ayant un père enseignant s'est élevée, dans le premier comme dans le second degré : pour les instituteurs / professeurs des écoles elle est passée de 6\% pour la cohorte née en 1930-1939 à I3\% pour la cohorte née en 1970-1979; pour les professeurs des collèges et lycées les proportions sont respectivement $9 \%$ et I $8 \%$. Lautorecrutement suppose une plus grande socialisation familiale au métier d'enseignant, préparant les jeunes à entrer «naturellement» dans le métier (Rayou et Van Zanten, 2004), à en adopter les codes, les valeurs et les réseaux de sociabilité, ainsi qu'à développer une rhétorique de la vocation fondée plus sur l'hérédité sociale que sur la sensation d'un rôle social à jouer (Périer, 2004). Le statut d'enseignant s'inscrit donc de plus en plus dans une logique de reproduction sociale, qui modifie le rapport qu'entretiennent les jeunes enseignants avec les générations précédentes: tandis que les jeunes des années 1970 ont adopté un nouveau statut social, s'élevant économiquement et culturellement par rapport à la posi- 
tion sociale de leurs parents, les jeunes des années 1990-2000 perpétuent plus souvent une situation que leurs parents ont acquise.

Cela ne résume cependant pas les dynamiques du recrutement social: au-delà de l'autorecrutement, elles sont distinctes selon le niveau d'enseignement, bien qu'elles aboutissent à un rapprochement des milieux d'origine. L’origine des professeurs du second degré s'est reportée vers le milieu de l'échelle sociale, notamment en raison de l'élévation de la proportion d'enfants d'enseignants et de professions intermédiaires. Si les pères ouvriers sont proportionnellement à peu près aussi nombreux quelle que soit la cohorte, la part des pères cadres et professions libérales a légèrement baissé au fil des cohortes, ce qui peut s'interpréter comme une moindre attirance pour le professorat des milieux favorisés, dont les enfants valorisent leurs diplômes dans d'autres professions, confirmant une tendance relevée par Claude Thélot (1994). En fait, c'est surtout la proportion de pères indépendants qui a décliné, ce qui est très vraisemblablement lié à des effets de structure, mais peut laisser supposer également un moindre intérêt pour le professorat de ces milieux.

Tandis que l'origine des enseignants du second degré s'est reportée vers le milieu de l'échelle sociale, celle des enseignants du premier degré s'est globalement élevée. Une approche de long terme montre en effet que, pour les enseignants du premier degré, sans considérer ici les différences selon le $\operatorname{sexe}^{22}$, les recrutements parmi les pères indépendants, les ouvriers et les employés ont globalement décliné dans la seconde moitié du $\mathrm{xx}^{\mathrm{e}}$ siècle, tandis qu’ont augmenté les recrutements parmi les pères cadres / professions libérales, professions intermédiaires ou enseignants. La féminisation de la population enseignante au cours du $\mathrm{Xx}^{\mathrm{e}}$ siècle y a fortement contribué (Delsaut, 1992)23, de même que la transformation de la structure des emplois au sein de la popu-

22 Une approche plus complète de la mobilité sociale des enseignants supposerait en effet de prendre en considération les dynamiques selon le genre ainsi que la profession de la mère, dont les travaux de Philippe Losego ont montré l'importance (1999).

23 Marlaine Cacouault-Bitaud (200I) insiste en outre sur l'absence de corrélation entre féminisation de l'enseignement et dévalorisation de ces professions, qui tient davantage selon elle aux réformes du système éducatif et à la place de l'école élémentaire, ainsi qu'à l'allongement des études et à l'augmentation du nombre de diplômés. 
lation active (Vallet et Degenne, 2000), bien que l'allongement du temps d'études ainsi que la sélectivité à l'entrée favorisent les candidats issus de milieux sociaux favorisés (Charles, 1988; Charles et Cibois, 2010). Il en ressort que, pour les cohortes nées jusqu’en 1950, la profession d' «instit» est associée à l'idée d’ascension sociale, tandis que, pour les cohortes puînées, elle est davantage synonyme de «déclassement $\gg{ }^{24}$. Certes, ces résultats ne disent pas quelle est la part des transformations de structure de la population active dans ces différences entre cohortes. Cependant, ils montrent que les caractéristiques sociales des enseignants ont changé, ce qui tend à distinguer le rapport à la profession, mais plus globalement le rapport au monde, des plus jeunes par rapport à celui des aînés (Spire, 20ı0).

Tableau 6: Profession du père des instituteurs / professeurs des écoles ${ }^{25}$

\begin{tabular}{|l|c|c|c|c|c|}
\hline & \multicolumn{5}{|c|}{ COHORTE DE NAISSANCE } \\
\hline \multicolumn{1}{|c|}{ PROFESSION DU PÈrRE } & $1930-1939$ & $1940-1949$ & $1950-1959$ & $1960-1969$ & $1970-1979$ \\
\hline Agriculteur, artisan, commerçant & 34 & 28,6 & 21,3 & 16,2 & 15,9 \\
\hline Cadre, profession libérale & 8,5 & 7,4 & 12,9 & 15,1 & 16 \\
\hline Enseignant & 5,8 & 7,1 & 7,4 & 12,9 & 13 \\
\hline Profession intermédiaire & 11,7 & 12,4 & 17,3 & 20,1 & 19,3 \\
\hline Employé & 17,7 & 17,4 & 16,1 & 15,6 & 16,7 \\
\hline Ouvrier TOTAL & 22,2 & 27,1 & 25 & 20 & 19,2 \\
\hline & 100 & 100 & 100 & 100 & 100 \\
\hline
\end{tabular}

24 Sauf si l'on considère, pour les enseignantes, la profession de la mère (Cacouault-Bitaud, 200 I).

25 Champ: Instituteurs/professeurs des écoles actifs identifiés à l'aide du code des professions (ont été regroupés ici les «instituteurs» (42II), «les directeurs d'école primaire ou maternelle» (42/4), et les «instituteurs de l'éducation spécialisée» (42/5)). La profession du père est identifiée selon la nomenclature des PCS à 2 chiffres, la catégorie «Enseignants» comprend les PCS 34 et 42 (les pères sans profession, chômeur ou dans d'autres situations ont été écartés de l'analyse). 
Tableau 7 : Profession du père des professeurs du second degré26

\begin{tabular}{|l|c|c|c|c|c|}
\hline & \multicolumn{5}{|c|}{ COHORTE DE NAISSANCE } \\
\hline \multicolumn{1}{|c|}{ PROFESSION DU Père } & $1930-1939$ & $1940-1949$ & $1950-1959$ & $1960-1969$ & $1970-1979$ \\
\hline Agriculteur, artisan, commerçant & 27,9 & 27,2 & 22,3 & 18 & 15,2 \\
\hline Cadre, profession libérale & 16,9 & 16,8 & 19,3 & 18,1 & 16,8 \\
\hline Enseignant & 9,4 & 9,2 & 8,8 & 13,3 & 18 \\
\hline Profession intermédiaire & 10,8 & 12 & 15,3 & 16,4 & 15,7 \\
\hline Employé & 15,1 & 16 & 14,9 & 13,8 & 14,4 \\
\hline Ouvrier TOTAL & 19,9 & 18,8 & 19,4 & 20,3 & 19,9 \\
\hline & 100 & 100 & 100 & 100 & 100 \\
\hline
\end{tabular}

[Tableau 6 et 7]. Source: Enquêtes Emploi (INSEE) 1984-2002 (microdonnées: Centre Maurice Halbwachs), calculs de l'auteur, effectifs pondérés.

\section{CONCLUSION}

Nous avons cherché à mesurer les transformations du statut social des enseignants français, de manière à considérer la façon dont des individus, recrutés à des périodes distinctes, construisent leur identité socioprofessionnelle. Des éléments présentés, il ressort que ceux qui appartiennent aux cohortes les plus récentes intègrent une profession dont la valeur sociale a décliné, et dont le temps d'études les distingue moins de l'ensemble des actifs qu'auparavant. Les jeunes enseignants ne bénéficient pas, dans une mesure semblable à ce qu’ont connu leurs aînés, du même prestige de leur profession, ni d'un privilège équivalent de considération sociale fondé sur le niveau d'études, pour reprendre deux des composantes du statut social proposées par Max Weber. En outre, les caractéristiques du conjoint se sont modifiées, dans le sens d’une moindre exceptionnalité de la position d'enseignant dans la société. Quant aux origines

26 Champ: Professeurs du second degré actifs identifiés à l'aide du code des professions (ont été regroupés ici les «professeurs agrégés et certifiés》 (34II), les «PEGC et maîtres auxiliaires de l'enseignement général» (422I) et «les enseignants du technique court» (4224)). La profession du père est identifiée selon la nomenclature des PCS à 2 chiffres, la catégorie «Enseignants» comprend les PCS 34 et 42 (les pères sans profession, chômeur ou dans d'autres situations ont été écartés de l'analyse). 
sociales, le «prestige de naissance » s'est globalement élevé et cette population compte davantage d'«héritiers » et de «déclassés». Par ailleurs, nous vérifions que les enseignants du premier et du second degré se sont rapprochés, du point de vue de leurs parcours universitaires comme de leurs parcours sociaux.

Nous faisons apparaître une relation en chiasme entre la valeur sociale et intellectuelle associée à la profession d'enseignant, qui tend à décliner, et l'origine sociale des individus, qui se porte davantage vers le milieu ou le haut de l'échelle sociale. Cette corrélation négative ne peut quaffecter l'expérience vécue par les enseignants, dans le sens d'un plus grand sentiment de déclassement. En outre, nous avons observé que les plus jeunes, souvent enfants d'enseignants, ont des comportements homogames plus importants que ceux de leurs aînés. Cela peut s'entendre comme une plus grande disposition des jeunes générations à choisir un pair pour conjoint, le sentiment de proximité sociale étant renforcé par l'éducation familiale reçue et les valeurs transmises. Cependant, si l'on garde à l'esprit que cette tendance se fait jour dans un contexte caractérisé par un faible sentiment de valorisation, elle peut s'interpréter comme une forme de fermeture sociale du groupe enseignant vis-à-vis des autres milieux, les contacts des enseignants se faisant avant tout avec d'autres enseignants. Une approche plus qualitative des relations entre pairs, réalisée par ailleurs (Farges, 2010), montre en l'occurrence les tensions dont celles-ci font l'objet.

À une époque charnière dans l'histoire de la population enseignante en France, marquée par de nombreux départs à la retraite, ces résultats montrent les modifications au cours des dernières décennies du regard social porté sur l'école à travers l'analyse des caractéristiques sociales de ses agents. Mais plus encore, ils mettent en évidence les enjeux du renouvellement générationnel: notre propos souligne la permanence du changement social et montre que des caractéristiques apparemment sans limite de durée peuvent n'être en réalité que l'ombre portée d'un certain «esprit du temps » et de la génération qui le porte (Mannheim, 1990 [1928]). La période d'entrée en activité marque durablement le parcours de vie d'un individu, or, les jeunes enseignants qui débutent actuellement connaissent des conditions d'entrée dans leur métier très éloignées de celles qu'ont rencontrées leurs collègues dans les années 1960-1970. Dès lors, il 
est bien question d'un changement d'époque pour la population enseignante, supposant d'autres façons d'être socialement, que portent déjà en eux les plus jeunes mais qui restent cependant encore peu visibles à l'échelle de la société française, en raison du temps long que suppose le mouvement des générations.

\section{BIBLIOGRAPHIE}

BALLION R., 1982, Les Consommateurs d'école, Paris, Stock.

BARATON M. et PERRONNET S., 2009, «Les départs en retraite des personnels de l'Éducation nationale entre 2000 et 2007 », in Ministère de l'Éducation nationale, DEPP, Note d'information, vol. 09.17.

BERGER I., 1957, «Le malaise socio-professionnel des instituteurs français (premiers résultats d'une enquête sur les instituteurs du Département de la Seine) », in International Review of Education, vol. 3, n 3, p. 335-346.

- et BENJAMIN R., 1964, L'Univers des instituteurs: étude sociologique sur les instituteurs et les institutrices du département de la Seine, Paris, Éditions de Minuit.

CACOUAULT-BITAUD M., 200I, «La féminisation d'une profession est-elle le signe d'une baisse de prestige?», in Travail, genre et sociétés, vol. 5, n I, p. $91-115$.

-, 2007, Professeurs... mais femmes. Carrières et vies privées des enseignantes du secondaire au $\mathrm{XX}$ e siècle, Paris, La Découverte.

CHAMBAZ C., MAURIN E. et TORELLI C., 1998, «L'évaluation sociale des professions en France: Construction et analyse d'une échelle des professions », in Revue Française de Sociologie, vol. 39, I, p. 177-226.

CHAPOULIE J-M., 1987, Les Professeurs de l'enseignement secondaire, un métier de classes moyennes, Paris, Éditions de la MSH.

- et MERLLIÉ D., 197I, Les Professeurs de l'enseignement du second degré, compte rendu d'enquête, Centre de Sociologie Européenne.

CHARLES F., 1988, Instituteurs: un coup au moral. Genèse d'une crise de reproduction, Paris, Ramsay. 
CHARLES F. et CIBOIS P., 20I0, «L'évolution de l'origine sociale des enseignants du primaire sur la longue durée: retour sur une question controversée», in Sociétés contemporaines, vol. 77, p. 31-56.

CHAUVEL L., 1998, Le Destin des générations, structure sociale et cohortes en France au $X x^{e}$ siècle, Paris, Presses Universitaires de France.

-, 2006a, «Générations sociales, perspectives de vie et soutenabilité du régime de protection sociale», in P. D. Culpepper, P. A. Hall et B. Palier (dir.), La France en mutation (1980-2005), Paris, Presses de Sciences Po, p. I57-196.

-, 2006b, Les Classes moyennes à la dérive, Paris, Éditions du Seuil.

COLLOVALD A., 1985, «La république du militant. Recrutement et filières de la carrière politique des députés socialistes en 1981 », in P. Birnbaum (dir.), Les Élites socialistes au pouvoir, 1981-1985, Paris, Presses Universitaires de France, p. II-52.

DELSAUT Y., 1992, La Place du maître: une chronique des Écoles normales d'instituteurs, Paris, L'Harmattan.

DUBET F., 2002, Le Déclin de l'institution, Paris, Éditions du Seuil.

DURKHEIM É., 1999 [1938], L'Évolution pédagogique en France, Paris, Presses Universitaires de France.

DURU-BELLAT M., 2006, L'Inflation scolaire, Paris, Éditions du Seuil.

FARGES G., 2010, Les Identités enseignantes à l'épreuve du temps, les transformations intergénérationnelles d'un groupe social (1970-2010), Doctorat de sociologie, Sciences Po, Observatoire Sociologique du Changement.

GERBOD P., 1965, La Condition universitaire en France au xixe siècle, Paris, Presses Universitaires de France.

GROSSEIN J-P., 2005, «Max Weber «à la française»? De la nécessité d'une critique des traductions », in Revue Française de Sociologie, vol. 46, n० 4, p. 883-904.

HAMON H. et ROTMAN P., 1984, Tant qu'il y aura des profs, Paris, Éditions du Seuil. LANTHEAUME F. et HÉLOU C., 2008, La Souffrance des enseignants, une sociologie pragmatique du travail enseignant, Paris, Presses Universitaires de France. 
LÉGER A., 1983, Enseignants du secondaire, Paris, Presses Universitaires de France.

LEMEL Y., 1991, Stratification et mobilité sociale, Paris, Armand Colin.

LOSEGO P., 1999, «La construction de la compétence professionnelle et sa mesure: le cas des futurs enseignants formés dans les IUFM», in Revue Française de Sociologie, vol. 41, n I, p. 139-169.

MANNHEIM K., 1990 [1928], Le Problème des générations, Paris, Armand Colin.

MENDRAS H., 1994, La Seconde Révolution française 1965-1984, Paris, Gallimard.

MICHEL P., 2007, «Concours de recrutement des professeurs des écoles, session 2006 », in Ministère de l'Éducation Nationale, de l'Enseignement Supérieur et de la Recherche, DEPP, Note d'information, vol. 06.20.

PÉRIER P., 2004, «Une crise des vocations? Accès au métier et socialisation professionnelle des enseignants du secondaire », in Revue Française de Pédagogie, vol. 147, p. 79-90.

PLAISANCE E., 1985, L'Échec scolaire. Nouveaux débats, nouvelles approches, Paris, Éditions du CNRS.

POUPEAU F., 2008, «Choix scolaires des familles», in A. Van Zanten (dir.), Dictionnaire de l'Éducation, Paris, Presses Universitaires de France, p. 325-329.

PROST A., 2004 [198I], Histoire de l'enseignement et de l'éducation, tome IV. Depuis 1930, Paris, Perrin.

RAYOU P. et VAN ZANTEN A., 2004, Enquête sur les nouveaux enseignants: changeront-ils l'école?, Paris, Bayard.

SAVOIE P., 2009, «Aux origines de la professionnalisation? La genèse du corps enseignant secondaire français », in Éducation et Sociétés, vol. 23, n I, p. 13-26.

SAWICKI F., 2008, «P.S. (Parti socialiste)», Encyclopaedia Universalis, p. 966-97I.

SINGLY (de) F. et POISSENOT C., 1996, «Les enseignants en couple», in Éducation et formations, vol. 46, p.93-105.

SPIRE A., 2010, «Les effets politiques des transformations du corps enseignant», in Revue Française de Pédagogie, vol. 170, p.61-72. 
THÉLOT C., 1994, «L'origine sociale des enseignants》, in Éducation et formations, vol. 37, p. 19-21.

VALETTE C., 2007, «Les concours de recrutement des personnels enseignants du second degré, session 2006 », in Ministère de l'Éducation Nationale, de l'Enseignement Supérieur et de la Recherche, DEPP, Note d'information, vol. 07.34.

VALLET L. A., 1996, «L'assimilation scolaire des enfants issus de l'immigration et son interprétation: un examen sur données françaises », in Revue Française de Pédagogie, vol. 117, p. 7-27.

- et DEGENNE A., 2000, «L'origine sociale des enseignants par sexe et niveau d'enseignement, évolution entre 1964 et 1997», in Éducation et formations, vol. 56, p. 33-40.

VAN DE VELDE C., 2008, Devenir adulte, sociologie comparée de la jeunesse en Europe, Paris, Presses Universitaires de France.

VINCENT G., 1967, Les Professeurs du second degré: contribution à l'étude du corps enseignant, Paris, Armand Colin.

WEBER M., 1976 [1922], Wirtschaft und Gesellschaft, Tübingen, Mohr Siebeck. -, 1995 [1956], Économie et Société, Les catégories de la sociologie, Paris, Pocket, trad. Freund Julien, Kamnitzer Pierre, Bertrand Pierre, et al. 